Palavras chave:

Pirólise

Biomassa

Carbonização

Composição química

Histórico:

Recebido 13/07/201 I

Aceito 15/12/2014

Keywords:

Pyrolysis

Biomass

Carbonization

Chemical composition

Correspondência: vassiasoares@gmail.com
Vássia Carvalho Soares', Maria Lúcia Bianchi², Paulo Fernando Trugilho², Juliana Höfler², Ariclene Júnior Pereira²

\section{ANÁLISE DAS PROPRIEDADES DA MADEIRA E DO CARVÃO VEGETAL DE HÍBRIDOS DE EUCALIPTO EM TRÊS IDADES}

RESUMO: Foram estudadas madeiras de híbridos de Eucalyptus grandis $x$ Eucalyptus urophylla em três diferentes idades. As características das madeiras e dos carvões produzidos, a partir dessas madeiras, bem como influência da madeira nas características dos carvões produzidos foram avaliadas. Foram realizadas análises químicas (extrativos, lignina, cinzas, análise elementar), densidade básica e poder calorífico superior - PCS nas madeiras. $O$ carvão foi produzido em forno tipo mufla, com taxa de aquecimento $\mathrm{I}, 67^{\circ} \mathrm{C} \cdot \mathrm{min}^{-1}$ e temperatura final de $450^{\circ} \mathrm{C}$. Para os carvões, foram realizadas análise imediata, análise elementar e rendimento gravimétrico do processo de pirólise. Foi observado um aumento nos valores de densidade, teor de extrativos, teor de carbono e relação $\mathrm{C} / \mathrm{H}$ da madeira com a maturidade da árvore. Outras características como teor de cinzas e relação $S / G$ tiveram seus valores diminuídos com a idade da árvore. Maior rendimento gravimétrico em carvão e em gases não-condensáveis foram encontrados no material mais maduro.

\section{PROPERTIES OF EUCALYPTUS WOOD HYBRIDS AND CHARCOAL AT THREE AGES}

ABSTRACT: In the present studied was investigated hybrids of Eucalyptus grandis $\mathrm{x}$ Eucalyptus urophylla in three different ages. Wood and charcoal characteristics as well as the influence of wood characteristics on the charcoals produced were evaluated. Chemical analyzes (extractives, lignin, ash, elemental analysis), basic density and calorific value PCS were performed for wood. The charcoals were produced in a muffle type furnace with a heating rate of $1.67^{\circ} \mathrm{C} \cdot \mathrm{min}^{-1}$ and an end temperature of $450{ }^{\circ} \mathrm{C}$. Volatile material content, ash content, fixed carbon content, elemental analysis and gravimetric yield of the pyrolysis process were performed for the charcoals. An increase in density values, extractives content, carbon content and $\mathrm{C} / \mathrm{H}$ rate were observed with the maturity of the tree. Other features such as ash content and S/G ratio decreased with the age of the tree. Greater gravimetric yield in charcoal and non-condensable gases were found in more mature materials.
DOI: 10.1590/01047760201521021294
I Instituto Federal Minas Gerais - Bambuí, Minas Gerais, Brasil

${ }^{2}$ Universidade Federal de Lavras - Lavras, Minas Gerais, Brasil 


\section{INTRODUÇÃO}

Um dos grandes problemas enfrentados pelas indústrias siderúrgicas é a heterogeneidade do carvão vegetal. Variações nas características do carvão vegetal são inerentes às condições do processo de carbonização e às características da madeira utilizada, tais como a espécie, as características químicas e a idade. As características da madeira se alteram com a idade, em função de alterações fisiológicas, podendo acarretar alteração na qualidade do carvão vegetal.

O efeito da idade sobre algumas características da madeira é bastante claro, como no caso do teor de cinza, que diminui com a idade da árvore (KUMAR et al., 2009; MORAIS, 2008; TRUGILHO et al., 1996), e a densidade básica aumenta com a idade da árvore (KUMAR et al., 20I0, 20I I; LEMENIH; BEKELE, 2004; MORAIS, 2008; RAYMOND, 2000; SILVA, 20II; SILVA et al., 2005; STURION et al., 1988; TRUGILHO et al., 1996; VITAL, 1984).

$O$ teor de lignina tende a diminuir com a idade da árvore (MORAIS, 2008; RAYMOND, 2000), pois as espécies mais jovens tendem a apresentar maior proporção de madeira juvenil, que é mais rica em lignina que a madeira madura (TRUGILHO et al., 1996). Entretanto, Silva et al. (2005), estudando Eucalyptus grandis com idades de 10, 14, 20 e 25 anos, observaram aumento no teor de lignina com a idade. Vários autores encontraram correlação positiva entre o teor de extrativos totais e a idade da árvore (MORAIS, 2008; RAYMOND, 2000; SILVA, 20II; SILVA et al., 2005) e atribuíram esse comportamento ao processo de cernificação, no qual os extrativos são incorporados ao cerne. Ao contrário de Silva et al. (2005), alguns autores obtiveram como resultado o aumento do teor de holocelulose com a idade da árvore (RAYMOND, 2000; SILVA, 20I I; TRUGILHO et al., 1996).

Lemenih e Bekele (2004) encontraram correlação negativa entre poder calorífico e idade, enquanto Kumar et al. $(2010,20 \mathrm{II})$ constataram que o poder calorífico aumenta em função da idade da árvore. Kumar et al. (20l I), estudando madeiras de híbridos de eucalipto de 2 a 6 anos, encontraram correlação positiva para poder calorífico e o teor de materiais voláteis, em função da idade da árvore. Em contrapartida, os teores de cinza e carbono fixo diminuíram com a maturidade. Esses autores consideraram a presença de cinza como fator redutor do poder calorífico.

Diante do exposto, objetivou-se neste trabalho, avaliar o comportamento das madeiras de híbridos de Eucalyptus grandis $x$ Eucalyptus urophylla em diferentes idades, mediante análise química e avaliar o carvão vegetal produzido.

\section{MATERIAL E MÉTODOS}

Foram utilizadas madeiras de híbridos de Eucalyptus grandis $x$ Eucalyptus urophylla em três idades distintas ( 3,5 e 7 anos), provenientes de plantio comercial localizado no estado de São Paulo. Foram retirados discos a I,30 m em relação à base da árvore. Para cada idade, foram amostradas nove árvores. Para a determinação da densidade básica foram utilizadas duas cunhas opostas de cada disco. As outras duas cunhas foram utilizadas para a análise química e preparo do carvão. A densidade básica da madeira foi avaliada utilizando o método de imersão, segundo a Norma NBR I I94 I (ASSOCIAÇÃO BRASILEIRA DE NORMAS TÉCNICAS - ABNT, 2003). $\mathrm{Na}$ madeira, foram realizadas as seguintes análises: extrativos (ASSOCIAÇÃO TÉCNICA BRASILEIRA DE CELULOSE E PAPEL - ABCP, 1968), lignina Klason (GOMIDE; DEMUNER, 1986), cinza (TAPPI TECHNICAL DIVISIONS AND COMMITTEES - TAPPI, 1998), relação siringil-guaiacil (LIM; DENCE, 1992) e poder calorífico superior (ABNT, 1983). A análise elementar (CHNS-O) foi realizada com $2,5 \mathrm{mg}( \pm 0,5)$ do material com granulometria menor que 200 mesh (madeira ou carvão), no equipamento CHNS-O Flash EA I I I 2 Series.

As carbonizações foram realizadas em forno elétrico adaptado com sistema de recuperação de gases. O dispositivo de entrada e saída de parâmetros foi informatizado. As cunhas de massa e tamanhos variáveis foram previamente secas em estufa, a $105^{\circ} \mathrm{C}$, e pirolisadas com taxa de aquecimento de aproximadamente I,67 ${ }^{\circ} \mathrm{C} \cdot \mathrm{min}^{-1}$ e temperatura final de $450^{\circ} \mathrm{C}$. Posteriormente à carbonização, foram determinados os rendimentos gravimétricos em carvão, em líquido pirolenhoso e em gases não-condensáveis. $A$ análise química imediata, com o objetivo de determinar os teores de umidade, materiais voláteis (TMV), cinza (TCZ) e, por diferença, o teor de carbono fixo (TCF), foi realizada de acordo com a norma NBR 81 I 2 da ABNT (1986). O poder calorífico superior do carvão também foi determinado, segundo a norma descrita anteriormente.

Para a análise do experimento foi utilizado o delineamento inteiramente casualizado, com três tratamentos (idades) e três repetições para cada idade. Para verificar a intensidade e a direção da relação linear entre a idade e as características da madeira e carvão vegetal, foi utilizada a análise de regressão linear, com intervalo de confiança de $95 \%$. 


\section{RESULTADOS E DISCUSSÃO}

Os valores médios da densidade básica e as características químicas da madeira e do carvão vegetal dos híbridos de Eucalyptus grandis x Eucalyptus urophylla nas três diferentes idades, encontram-se na Tabela I.

TABELA 1 Valores médios da densidade básica e características químicas da madeira. Rendimentos gravimétricos e características químicas do carvão.

TABLE 1 Average values of basic density and chemical characteristics of wood. Gravimetric yield and chemical characteristics of charcoal.

\begin{tabular}{|c|c|c|c|}
\hline \multicolumn{4}{|c|}{ Madeira } \\
\hline & 3 anos & 5 anos & 7 anos \\
\hline $\mathrm{d}\left(\mathrm{g} \cdot \mathrm{cm}^{-3}\right)$ & 0,37 & 0,44 & 0,54 \\
\hline $\operatorname{Lig}^{*}(\%)$ & 21,52 & 21,52 & 21,97 \\
\hline Extrativos (\%) & 8,26 & 9,44 & 9,43 \\
\hline TCZ (\%) & 0,41 & 0,31 & 0,18 \\
\hline PCS $\left(\mathrm{kcal} \cdot \mathrm{kg}^{-1}\right)$ & 4454 & 4547 & 4385 \\
\hline C (\%) & 43,97 & 44,49 & 45,60 \\
\hline $\mathrm{O}(\%)$ & 49,22 & 48,75 & 47,75 \\
\hline $\mathrm{C} / \mathrm{H}$ & 7,12 & 7,20 & 7,39 \\
\hline$S / G$ & 3,50 & 3,20 & 2,30 \\
\hline \multicolumn{4}{|c|}{ Carvão vegetal } \\
\hline & 3 anos & 5 anos & 7 anos \\
\hline RGC (\%) & 31,61 & 30,55 & 33,06 \\
\hline RLP (\%) & 40,22 & 44,19 & 38,55 \\
\hline RGNC (\%) & 28,18 & 25,26 & 28,39 \\
\hline TMV (\%) & 20,08 & 18,85 & 22,82 \\
\hline TCF (\%) & 78,39 & 80,29 & 76,85 \\
\hline TCZ (\%) & 1,53 & 0,87 & 0,33 \\
\hline PCS $\left(\mathrm{kcal} \cdot \mathrm{kg}^{-1}\right)$ & 7062 & 7215 & 7193 \\
\hline C (\%) & 81,28 & 81,60 & 79,81 \\
\hline $\mathrm{O}(\%)$ & 15,16 & 14,89 & 16,60 \\
\hline $\mathrm{C} / \mathrm{H}$ & 24,84 & 24,82 & 23,43 \\
\hline
\end{tabular}

*propriedades sem diferença significativa entre as idades $d=$ densidade básica, $\mathrm{Lig}=$ lignina, $\mathrm{TCZ}=$ teor de cinza, PCS = poder calorífico superior, $S / G$ = relação siringil-guaiacil, $R G C=$ rendimento gravimétrico em carvão vegetal, RLP = rendimento gravimétrico em líquido pirolenhoso, RGNC = rendimento gravimétrico em gases não-condensáveis, $\mathrm{TMV}=$ teor de materiais voláteis, $\mathrm{TCF}=$ teor de carbono fixo.

$\mathrm{Na}$ madeira, observou-se que o efeito da idade não foi significativo para os teores de lignina. Os teores médios de lignina estão próximos aos valores encontrados por outros autores para o gênero Eucalyptus (Brito et al., 1983; Trugilho et al., 1996, 2007).

\section{Densidade básica da madeira}

$\mathrm{Na}$ Figura I, observa-se a relação funcional observada entre a densidade básica e a idade da árvore. Valores semelhantes foram encontrados por outros autores (RIBEIRO; ZANI-FILHO, 1993) ao estudarem a densidade básica da madeira de diferentes espécies de eucalipto, aos 6 anos de idade. Com a maturidade da árvore, observou-se um aumento na densidade básica da madeira. Segundo Vital (1984), essa tendência é consequência do aumento da espessura da parede celular e da diminuição da largura das células.

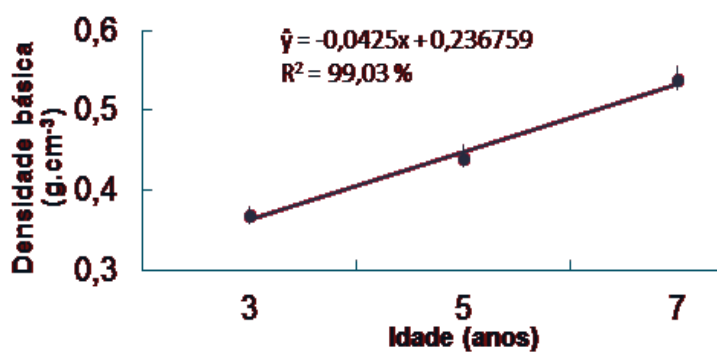

FIGURA 1 Densidade da madeira em função da idade da árvore.

FIGURE 1 Wood density in function of tree age.

\section{Propriedades químicas da madeira: teor de cinza e extrativos}

As relações entre o teor de extrativos e cinza em função da idade da madeira estão apresentados na Figura 2. O aumento no teor de extrativos em função da idade do material lenhoso pode ser justificado, em razão do início do processo de cernificação, no qual ocorre a transformação do alburno em cerne. Esse processo é caracterizado pela morte de células e alterações na constituição química das mesmas. Higuchi (1997) afirma que o aumento no conteúdo de extrativos, especialmente de polifenóis, deve-se à alteração da atividade celular ou fisiológica. Morais (2008) observou a mesma tendência para o teor de extrativos em função da idade da madeira de clones de eucalipto e afirmou, ainda, que o processo de cernificação se intensificou, a partir dos cinco anos, para o material estudado.

O teor de cinza diminuiu significativamente com a maturidade da árvore, provavelmente em decorrência da diminuição da atividade fisiológica da árvore com a idade, exigindo, portanto, menor presença de componentes minerais. A mesma tendência foi observada por Morais (2008). 

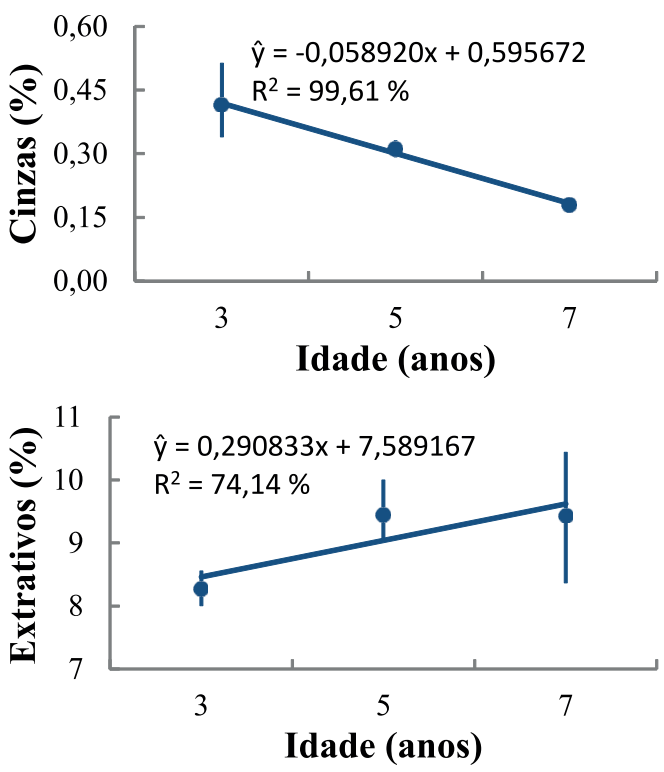

FIGURA 2 Relação entre os teores de cinza e extrativos e a idade da madeira.

FIGURE 2 Relationship of ash and extractives content and the wood age.

\section{Relação entre o teor de lignina siringila e guaiacila (S/G)}

$\mathrm{Na}$ Figura 3, está representada a relação S/G da lignina em função da idade da árvore. Apesar de não haver diferença quantitativa significativa entre os teores de lignina nas diferentes idades, o tipo de lignina presente no material mudou. Observou-se diminuição na relação $\mathrm{S} / \mathrm{G}$, o que significa um aumento na quantidade do grupo guaiacil na macromolécula, com o aumento da idade da árvore. A mesma tendência foi observada por Morais (2008). Para madeiras destinadas à produção de carvão vegetal, deve-se procurar por materiais genéticos com menores relações $S / G$, uma vez que o grupo guaiacil é mais preservado no processo de pirólise. O grupo siringil possui um grupo metoxila a mais do que o guaiacil, tornando-se mais reativo, ou seja, mais facilmente oxidado durante a pirólise.

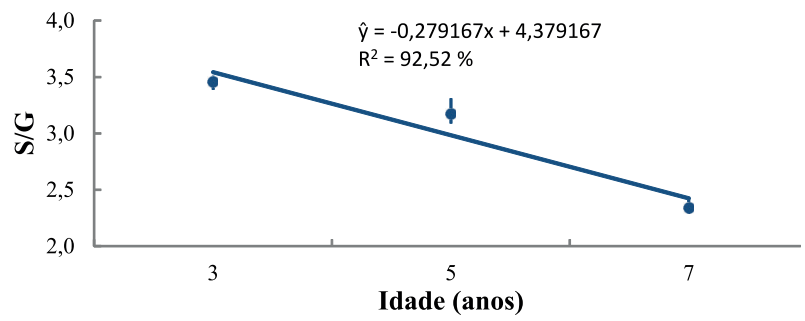

FIGURA 3 Teor de lignina siringila-guaiacila em função da idade da madeira.

FIGURE 3 Syringyl-guaiacyl rate in relation to wood age.
Ainda, a ausência de um grupo metoxila na estrutura da lignina possibilita a formação de ligações C-C com outra unidade guaiacil, promovendo a condensação de anéis aromáticos. $O$ processo de condensação é evidenciado em estudo realizado por Piló-Veloso et al. (1993). A maior eletronegatividade dos átomos de oxigênio ocasiona uma carga parcial negativa ao redor desse átomo, tornando as ligações C-O-C mais reativas e, portanto, menos estáveis que as ligações C-C (SOLOMONS, 1996). Assim, supõe-se que as ligações $C-C$ são mais resistentes à degradação química que as C-O-C, o que dificulta a quebra da molécula de lignina. Pode-se afirmar, portanto, que a lignina formada principalmente por grupo do tipo guaiacil será menos reativa que aquela formada pelo grupo siringil.

\section{Rendimentos gravimétricos da pirólise}

$\mathrm{Na}$ Figura 4, são apresentados os valores dos rendimentos gravimétricos em função da idade. $O$ maior valor de rendimento gravimétrico em carvão vegetal (RGC) foi encontrado no material mais maduro.
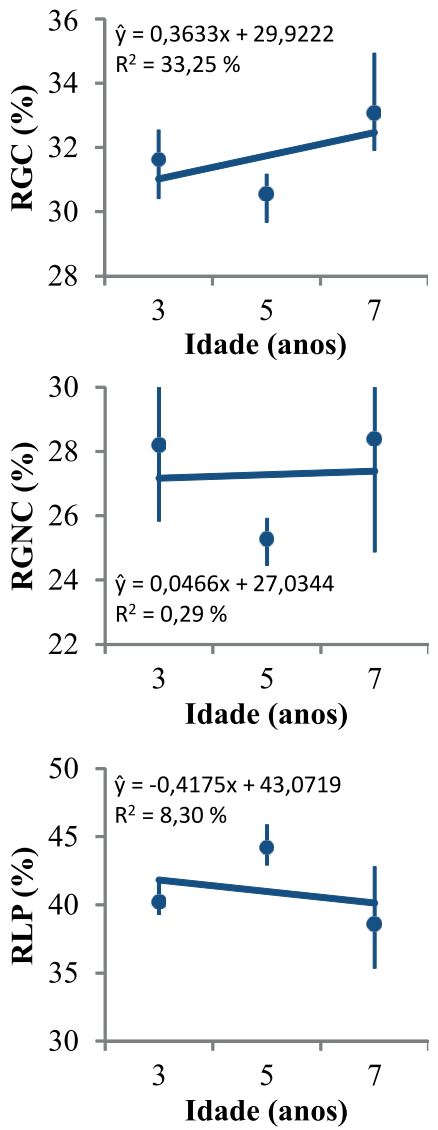

FIGURA 4 Rendimentos gravimétricos da carbonização em função da idade da madeira.

FIGURE 4 Charcoal gravimetric yields in relation to wood age. 
A composição química e as demais propriedades do carvão vegetal são fortemente influenciadas pela temperatura final da carbonização e taxa de aquecimento. No presente estudo, essas duas variáveis foram as mesmas para todas as carbonizações. Dessa forma, variações no RGC, RGNC e RLP podem ser atribuídas somente às características químicas e físicas (tamanho da amostra) do material a ser carbonizado.

Em estudos realizados por diversos autores (BRITO; BARRICHELO, 1977; OLIVEIRA et al., 1989) constatou-se que $\circ$ teor de lignina está intimamente relacionado ao rendimento gravimétrico em carvão vegetal. Entretanto, no presente estudo, não houve diferença significativa entre os teores de lignina nas três idades estudadas. Assim, o RGC pode ter sido influenciado pelo tipo de lignina presente na madeira e relação $\mathrm{C} / \mathrm{H}$. A variação existente nos rendimentos gravimétricos em carvão, gases não-condensáveis e líquido pirolenhoso não pode ser explicada exclusivamente pela idade da madeira.

\section{Análise imediata do carvão}

$\mathrm{Na}$ Figura 5, apresenta-se a relação entre os parâmetros analisados na análise imediata do carvão vegetal - teor de materiais voláteis (TMV), teor de carbono fixo (TCF) e teor de cinza (TCZ) - e a idade do material vegetal. A variação existente nos teores de materiais voláteis e teores de carbono fixo não pode ser explicada exclusivamente pela idade da madeira. $O$ teor de cinza apresentou a mesma tendência observada na madeira, ou seja, de diminuir em função do aumento da idade da árvore.

\section{Poder calorífico da madeira e do carvão}

Os valores encontrados para o poder calorífico da madeira e do carvão vegetal estão apresentados na Figura 6. As amostras de madeira estudadas apresentaram poder calorífico com valores entre 4.200 e $4.800 \mathrm{kcal} \cdot \mathrm{kg}^{-1}$. Para o carvão vegetal, foram encontrados valores entre 6.684 e $7.50 \mathrm{I} \mathrm{kcal} \cdot \mathrm{kg}^{-1}$. Observa-se, em função da idade, ligeira diminuição do PCS da madeira e ligeiro aumento no PCS do carvão.

O teor de oxigênio elementar se reduz para aproximadamente $30 \%$ do teor original após a pirólise (Tabela 1). Dessa forma, ligações oxigenadas não estão presentes em grande quantidade no carvão. $\mathrm{Na}$ madeira, o oxigênio apresenta, principalmente, ligações simples com hidrogênio $(\mathrm{H}-\mathrm{O})$ ou carbono (C-O). As
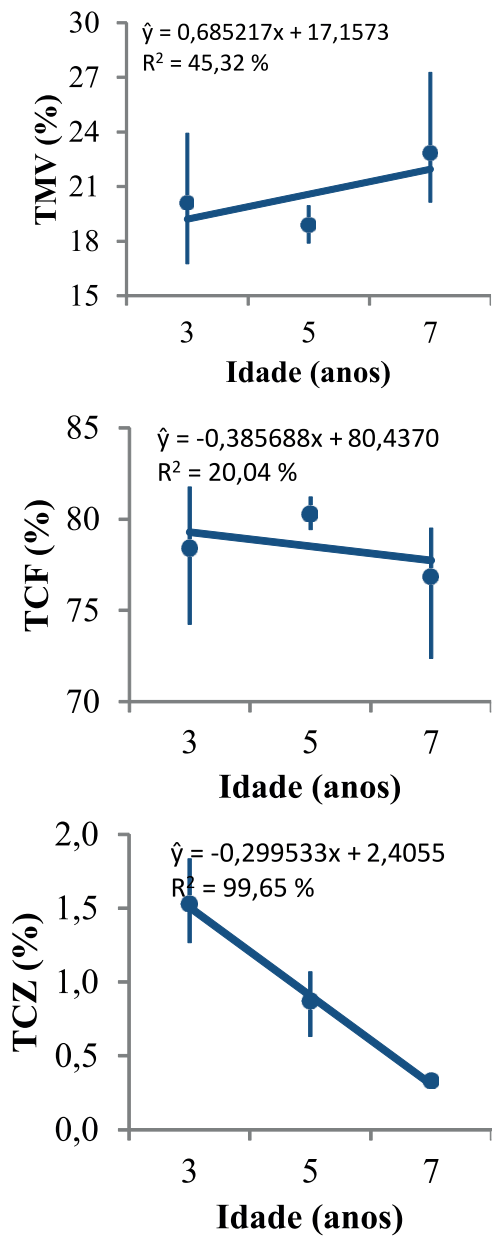

FIGURA 5 Análise imediata do carvão em função da idade da madeira.

FIGURE 5 Chemical composition of charcoal in relation to wood age.

(a)

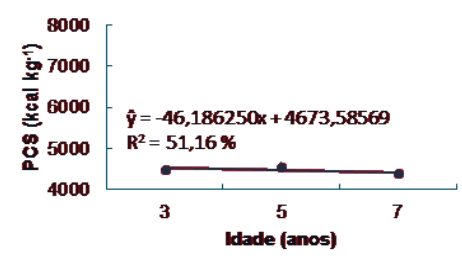

(b)

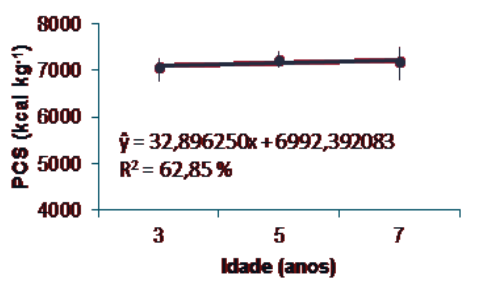

FIGURA 6 Poder calorífico superior (a) da madeira e (b) do carvão em função da idade

FIGURE 6 Calorific value of (a) wood and (b) charcoal in relation to wood age 
energias de ligação são, respectivamente, $463 \mathrm{~kJ} \cdot \mathrm{mol}^{-1}$ e $360 \mathrm{~kJ} \cdot \mathrm{mol}^{-1}$. Durante a pirólise, compostos contendo carbonos saturados (ligação simples C-C), tais como celulose e hemiceluloses, são degradados. A energia de ligação entre dois carbonos é da ordem de $348 \mathrm{~kJ} \cdot \mathrm{mol}^{-1}$ para ligações C-C e $518 \mathrm{~kJ} \cdot \mathrm{mol}^{-1}$ para ligações $C=C$, em compostos aromáticos (ATKINS; JONES, 2006). $\mathrm{Na}$ madeira, existe maior quantidade de compostos contendo ligações simples e que possuem menor energia. No carvão vegetal, em decorrência da presença de compostos aromáticos e, portanto, mais insaturados, a energia armazenada é maior. Assim, o poder calorífico do carvão vegetal é maior que o da madeira, pois, durante o processo de pirólise, parte dos componentes menos energéticos da madeira foi degradada, ficando preservadas as estruturas que contêm carbonos insaturados, tais como os anéis aromáticos presentes na estrutura da lignina.

\section{CONCLUSÕES}

A densidade, teor de extrativos, teor de carbono e relação $\mathrm{C} / \mathrm{H}$ da madeira aumentaram com a maturidade da árvore. $O$ teor de cinza, relação $\mathrm{S} / \mathrm{G}$, bem como o teor de cinza e relação $\mathrm{C} / \mathrm{H}$ do carvão diminuíram com a idade da árvore. $\mathrm{O}$ carvão apresentou maior poder calorífico superior que a madeira, em razão da maior aromaticidade do material carbonizado. Os maiores valores de RGC e RGNC foram encontrados no material mais maduro.

\section{AGRADECIMENTOS}

Os autores agradecem à CAPES, CNPq, FAPEMIG e LCP - UFV.

\section{REFERÊNCIAS}

ASSOCIAÇÃO BRASILEIRA DE NORMAS TÉCNICAS.

NBR 8I I 2: carvão vegetal: análise imediata. Rio de Janeiro, 1986. $8 \mathrm{p}$.

ASSOCIAÇÃO BRASILEIRA DE NORMAS TÉCNICAS.

NBR 8633: carvão vegetal: determinação do poder calorífico. Rio de Janeiro, 1983. I 3 p.

ASSOCIAÇÃO BRASILEIRA DE NORMAS TÉCNICAS.

BR I 194I: madeira, determinação da densidade básica. Rio de Janeiro, 2003. 6 p.
ASSOCIAÇÃO TÉCNICA BRASILEIRA DE CELULOSE E PAPEL. Normas de ensaio. São Paulo, 1968. 6 p.

ATKINS, P; JONES, L. Princípios de química: questionando a vida moderna e o meio ambiente. 3 . ed. Porto Alegre: Bookman, 2006. 968 p.

BRITO, J. O.; BARRICHELO, L. E. G. Correlações entre características físicas e químicas da madeira a produção de carvão: I., densidade e teor de lignina da madeira de eucalipto. IPEF, Piracicaba, v. 14, p. 9-20, 1977.

BRITO, J. O.; BARRICHELO, L. E. G.; SEIXAS, F; MIGLIORINI, A. J.; MURAMOTO, M. C. Análise da produção energética e de carvão vegetal de espécies de eucalipto. IPEF, Piracicaba, n. 23, p. 53-56, abr. 1983.

GOMIDE, J. L.; DEMUNER, B. J. Determinação do teor de lignina em material lenhoso: método Klason modificado. O Papel, São Paulo, v. 47, n. 8, p. 3638, 1986.

HIGUCHI, T. Biochemistry and molecular biology of wood. Berlin: Springer-Verlag, 1997. 362 p.

KUMAR, R.; CHANDRASHEKAR, N.; PANDEY, K. $K$. Fuel properties and combustion characteristic of Lantana Camara and Eupatorium spp. Current Science, Columbus, v. 97, n. 6, p. 930-934, Dec. 2009.

KUMAR, R. et al. Effect of tree-age on calorific value and other fuel properties of Eucalyptus hybrid. Journal of Forestry Research, Amsterdam, v. 2I, n. 4, p. 5|4-516, Dec. 2010.

KUMAR, R. et al. Study of age and height wise variability on calorific value and other fuel properties of Eucalyptus hybrid, Acacia auriculaeformis and Casuarina equisetifolia. Biomass and Bioenergy, Oxford, v. 35, n. 3, p. I339-I344, Mar. 201 I.

LEMENIH, M.; BEKELE, T. Effect of age on calorific value and some mechanical properties of three Eucalyptus species grown in Ethiopia. Biomass and Bioenergy, Oxford, v. 27, n. 3, p. 223-232, Sept. 2004.

LIM, S. Y.; DENCE, C. W. Methods in lignin chemistry. Berlin: Springer-Verlag, 1992. 578 p. 
MORAIS, P. H. D. Efeito da idade da madeira de eucalipto na sua química e polpabilidade, e branqueabilidade e propriedades físicas da polpa. 2008. 79 p. Dissertação (Mestrado em Agroquímica) - Universidade Federal de Viçosa, Viçosa, MG, 2008.

OLIVEIRA, E. de; VITAL, B. R.; VALENTE, O. F.; GOMIDE, J. L. Efeito da qualidade da madeira sobre o rendimento e qualidade do carvão de Eucalyptus grandis. Revista Árvore, Viçosa, v. 13, n. I, p. 8597, 1989.

PILÓ-VELOSO, D.; NASCIMENTO, E. A.; MORAIS, S. A. L. Isolamento e análise estrutural de ligninas. Química Nova, São Paulo, v. I6, n. 5, p. 435-448, 1993.

RAYMOND, C. A. Tree breeding issues for solid wood products. In: THE FUTURE OF EUCALYPTUS FOR WOOD PRODUCTS, I., 2000, Launceston. Proceedings... Launceston: IUFRO, 2000. p. 265-270.

RIBEIRO, F. A.; ZANI-FILHO, J. Variação da densidade básica da madeira em espécies/procedências de Eucalyptus spp. IPEF, Piracicaba, n. 46, p.76-85, jan./ dez. 1993.

SILVA, J. C.; MATOS, J. L. M.; OLIVEIRA, J. T. S.; EVANGELISTA, W. V. Influência da idade e da posição ao longo do tronco na composição química da madeira de Eucalyptus grandis Hill ex. Maiden. Revista Árvore, Viçosa, v. 29, n. 3, p. 455-460, 2005.
SILVA, M. G. S. Produtividade, idade e qualidade de madeira de Eucalyptus destinada à produção de polpa celulósica branqueada. 2011. 94 p. Dissertação (Mestrado em Tecnologia de Produtos Florestais) - Escola Superior de Agricultura "Luiz de Queiroz", Piracicaba, 20II.

SOLOMONS, T. W. G. Química orgânica I. Rio de Janeiro: LTC, 1996.

STURION, J. A.; PEREIRA, J. C. D.; CHEMIN, M. S. Qualidade da madeira de Eucalyptus viminalis para fins energéticos em função do espaçamento e idade de corte. Boletim de Pesquisa Florestal, Colombo, $\mathrm{n}$. I6, p. 55-59, 1988.

TAPPI TECHNICAL DIVISIONS AND COMMITTEES. TAPPI test methods. Atlanta, 1998. 460 p.

TRUGILHO, P. F.; BIANCHI, M. L.; ROSADO, S. C. S.; LIMA, J. T. Qualidade da madeira de clones de espécies e híbridos naturais de Eucalyptus. Scientia Forestalis, Piracicaba, n. 73, p. 55-62, mar. 2007.

TRUGILHO, P. F.; LIMA, J. T.; MENDES, L. M. Influência da idade nas caracteristicas fisico-quimicas e anatômicas da madeira de eucalyptus saligna. Cerne, Lavras, v. 2, n. I, p. 94-II I, 1996.

VITAL, B. R. Métodos de determinação de densidade da madeira. Viçosa, MG: SIF, 1984. 21 p. (Boletim Técnico, I). 
